\title{
SCIENTIFIC PROCEEDINGS
}

Abstracts of Communications.

One hundred tenth meeting.

New York Post Graduate Medical School, New York City, November 17, 1920. President Calkins in the chair.

25 (1607)

Preliminary experiments with the fat-soluble vitamine (vitamin A). By H. C. Sherman, F. L. Macleod and M. M. Kramer.

[From the Laboratory of Food Chemistry, Columbia University.]

The term "fat soluble vitamine" or "vitamin A" is here employed to designate the substance or substances occurring in butter fat, egg fat, codliver oil and elsewhere by virtue of which growth is promoted when the diet is otherwise adequate, and the characteristic eye disease, noticed especially in rats by Osborne and Mendel, is prevented and may often be cured. If, as indicated by some recent observations, especially those of Hess, the relations of butter fat and codliver oil to rickets are so different as to suggest that their vitamines are different substances, it becomes conceivable that more than one substance having growth-promoting and "antixerophthalmic" properties may be embraced under the one term "fat-soluble vitamine" or "vitamin A" as now used.

I. Distribution of the Substance or Substances "Vitamin A" between the Fatty and Aqueous Phases in Milk.-Several years ago McCollum stated in a brief note that fat soluble A is about thirty times more soluble in fat than in water, in which case skimmed milk will contain about half as much of this vitamine as whole milk. On the other hand, Mellanby, studying experimental rickets in puppies, and Hess and Unger in their studies of the clinical rôle of the fat soluble vitamine, appear to have assumed that their experimental diets could contain considerable amounts 
of skimmed milk, either in fluid or solid form, and still be nearly devoid of the fat soluble vitamine. According to our experience, skimmed milk contains a very significant amount of fat soluble vitamine, probably about half as much as whole milk as McCollum's brief statement would imply.

Our experimental evidence of the presence of significant amounts of fat soluble vitamine or "vitamin A" in skimmed milk is two-fold. (I) Young rats placed at weaning upon a diet in which dried skimmed milk was the sole source of vitamines have grown steadily (though at less than the maximum rate) for three months or more, trebling their body weights and remaining free from eye disease and in good general condition. Such results in rats of this age can be obtained only on diets furnishing significant amounts of "vitamin A." (2) Rats which had been brought to the typical condition of declining body weight and characteristic eye disease due to deficiency of fat soluble vitamine in their food have been cured by the feeding of skimmed milk powder. [A third type of experiment may be mentioned which, while it would not be conclusive alone, affords interesting confirmation. Rats which had failed to grow upon a diet of white bread, grew with extraordinary rapidity for some time (though not to full adult size) when the bread was supplemented by dried skimmed milk only. The latter of course supplemented the bread in several ways, but unless the skimmed milk had furnished important amounts of fat soluble vitamine such rapid and extensive growth would hardly have been possible.]

2. Fat-soluble Vitamine in Growth and Reproduction.-The results of our experiments are entirely consistent with previous findings that fat soluble vitamine is essential to growth and indicate further that the proportion of this vitamine in the food may have quite as striking an influence upon reproduction as upon growth.

3. Storage of Fat-soluble Vitamine in the Body.-While a moderate concentration of this vitamine in a diet excellent in all other respects suffices to support normal growth, and a more liberal supply of the vitamine may not then affect the growth rate, it appears that a surplus of the vitamine above the immediate needs of growth or maintenance may be largely stored in the body and subsequently utilized to meet the needs of reproduction and 
lactation, or to carry the animal over a period of subsistence upon foods deficient in this vitamine. Animals kept under like conditions upon the same diet deficient in fat soluble vitamine show different survival periods according as their previous diet was rich or poor in this substance. Our results suggest that it may be largely because of previous storage of this vitamine that adult animals seem less dependent upon it than do young of the same species.

4. Heat Destruction of the Fat Soluble Vitamine.-Dry heating at a temperature of $100^{\circ} \mathrm{C}$. with free access of air, only very slowly destroyed fat soluble vitamine. The results thus far obtained emphasize the importance of taking full account of the time as well as the temperature of heating, and of the initial concentration of the vitamine in the food, as well as of the opportunity for previous storage of the vitamine by the test animal.

Experiments upon these four problems are being continued.

26 (I608)

A new diet for gastric ulcer.

By WARReN Coleman.

[Department of Medicine, University and Bellevue Hospital Medical College, New York City.]

From the dietetic standpoint the two main indications for the treatment of gastric ulcer are:

I. To protect the ulcer from mechanical and chemical injury, and

2. To maintain the nutrition of the patient at a level which will favor the healing of the ulcer.

In the author's opinion, none of the diets hitherto employed in the treatment of gastric ulcer fulfills these indications: in none of them do the therapeutic properties of food appear to have received adequate consideration. In general, the frequent administration of small or moderate quantities of milk constitutes the basis of diets for gastric ulcer. Even admitting the economic quality of the secretion called forth by milk, its hourly or two- 